\title{
CAUSAS E TRATAMENTO DA ANORGASMIA FEMININA: UMA REVISÃO DA LITERATURA.
}

Categoria: Clínico

Instituição: Centro Universitário São Camilo

Autores: Santos, R.R., Matumoto, A.N., Moreti, G.V., Rodrigues, G., Pasqualino, J.V.P., Mattos, S.R.

Orientadora: Pereira, M.M.

Dados do autor principal:

Endereço: Rua Cipriano Barata, 1638, apartamento 24

Telefone: (19) 99696-9774

Email: santos.rafa@,icloud.com 
CAUSAS E TRATAMENTO DA ANORGASMIA FEMININA: UMA REVISÃO DA LITERATURA.

Categoria: Clínico

Descritores: female anorgasmia, treatment 


\section{CAUSAS E TRATAMENTO DA ANORGASMIA FEMININA: UMA REVISÃO DA LITERATURA.}

Autores: Santos, R.R., Matumoto, A.N., Moreti, G.V., Rodrigues, G., Pasqualino, J.V.P., Mattos, S.R.

Orientadora: Pereira, M.M.

Email do autor principal: santos.rafa@icloud.com

Instituição: Centro Universitário São Camilo

INTRODUÇÃO: O orgasmo é uma sensação de prazer que melhora a qualidade de vida e a relação com o parceiro. Diversas situações podem levar à anorgasmia, exigindo uma identificação precoce e abordagens eficazes. Logo, esse estudo aborda suas possíveis causas e tratamentos. METODOLOGIA: Foi realizada uma revisão sistemática na base PubMed usando: "female anorgasmia AND treatment" de 2000 a 2021, obtendo 76 artigos. Incluiu-se os relacionados à questão norteadora e, ao aplicar os critérios de exclusão (revisões e fuga do tema), obteve-se 24 estudos. RESULTADOS: A cirurgia pélvica foi relacionada à anorgasmia em $11,8 \%$ das pacientes de um estudo transversal; uma coorte com 6 pacientes submetidas à genitoplastia indicou anorgasmia em todas e um relato de caso expôs anorgasmia temporária após embolização da artéria uterina. Entretanto, notou-se melhora da anorgasmia em 37 mulheres após correção de prolapso de órgãos pélvicos. Quanto aos efeitos colaterais de medicamentos, em um estudo a anorgasmia teve efeito predominante na paroxetina em relação ao escitalopram (26.2\% vs. 5.9\%); e, em outro estudo, a anorgasmia foi relatada por 5,9\% das mulheres em uso da reboxetina vs escitalopram (39\%). Já a fluoxetina, ao ser administrada na síndrome pré-menstrual, foi associada a 10\% de anorgasmia em um estudo com 30 mulheres. Todos os estudos relacionados a anticonvulsivantes foram relatos de caso, em um deles, 7 pacientes desenvolveram anorgasmia após uso de topiramato para enxaqueca, resolvendo o quadro com a redução/descontinuação do tratamento. Outras classes apareceram com menos frequência: antipsicóticos e implante hormonal. Em relação ao tratamento, foi descrito que o sildenafil e o vardenafil são eficazes para a reversão da anorgasmia, e a ciproeptadina, quando administrada em usuários de antidepressivos, conseguiu reduzir ou reverter a anorgasmia causada por estes. Além dessas classes, um estudo randomizado com 57 pacientes histerectomizadas relatou melhora importante da anorgasmia utilizando-se estrogênio oral e tópico ( $\mathrm{p}<0,05)$. CONCLUSÃO: A anorgasmia é uma afecção multifatorial que envolve tanto fatores sociais, quanto efeitos medicamentosos e intervenções cirúrgicas. Logo, baseado em evidências, os tratamentos vistos como satisfatórios foram: uso de agentes inibidores da fosfodiesterase, anti-histamínicos de primeira geração, terapia estrogênica e fitoterápicos associados ao encaminhamento interdisciplinar. DESCRITORES: female anorgasmia, treatment. 


\section{CAUSAS E TRATAMENTO DA ANORGASMIA FEMININA: UMA REVISÃO DA LITERATURA.}

\section{INTRODUÇÃO}

O orgasmo é uma sensação de intenso prazer que cria um estado de consciência alterado acompanhado por musculatura circunvaginal estriada pélvica e contrações uterinas, anais e miotonia que resolve a vasoconstrição induzida sexualmente. Esse ato melhora a autoestima, o humor, a qualidade de vida e a relação com o parceiro sexual. Dessa maneira, a anorgasmia - incapacidade de atingir o orgasmo durante a relação sexual pode afetar a qualidade de vida da mulher como um todo.

De maneira geral, distúrbios do orgasmo em mulheres, seja ele por atraso persistente/ recorrente ou ausência de orgasmo, afetam até 25\% das mulheres. Entre o amplo grupo de disfunções sexuais, encontramos a anorgasmia. Essa disfunção é tida pela incapacidade em atingir o orgasmo durante o ato sexual.

A anorgasmia pode ocorrer desde problemas psicológicos ao uso de medicamentos, principalmente antidepressivos. Dessa maneira, muitas vezes é necessária uma abordagem multiprofissional. Contudo, o tabu sobre esse assunto, ainda persiste na sociedade, o que reduz a procura pelo tratamento ou a compreensão do que está a levando a esse estado. Logo, esse estudo visa valorizar esse problema, abordando suas possíveis causas e tratamentos.

\section{OBJETIVO}

O presente estudo visa revisar fatores relacionados à anorgasmia e seus possíveis tratamentos, tanto medicamentosos como comportamentais. 


\section{METODOLOGIA}

Foi realizada uma revisão sistemática da literatura na base de dados PubMed utilizando os termos: "female anorgasmia AND treatment" no período de 2000 a 2021 . Um total de 76 artigos foram encontrados. Destes foram incluídos estudos que se correspondiam à questão norteadora dessa revisão e após aplicação dos critérios de exclusão: artigos de revisão e fuga do tema; foram utilizados 24 estudos originais. 


\section{RESULTADOS}

A partir da análise dos estudos incluídos na revisão, dividiu-se os resultados em quatro tópicos de grande importância para a compreensão da anorgasmia na prática clínica e acompanhamento das pacientes afetadas por essa queixa. Tais tópicos incluem cirurgias, efeitos colaterais de medicamentos, tratamento medicamentoso e não medicamentoso da anorgasmia.

Em relação às cirurgias, um estudo transversal ${ }^{1}$ que avaliou através de questionários (antes e após 3 meses), 37 mulheres sexualmente ativas submetidas à cirurgia de correção de prolapso de órgãos pélvicos, apresentou melhora nos escores Golombok Rust Inventory of Sexual Satisfaction (GRISS) após 3 meses da cirurgia [4,5 $\pm 2,6$ para 3,6 $\pm 2,2, p<0,001$; diminuição média de 0,9 , intervalo de confiança de 95\% (IC) 0,36-1,36] e nas subescalas de evitação do parceiro, vaginismo, não comunicação, insatisfação e anorgasmia. Além disso, houve melhora dos escores do Pelvic Floor Distress Inventory (PFDI) de $122 \pm 53$ no início do estudo para $28 \pm 31$ em 3 meses.

Em contraposição, piores desfechos em relação à anorgasmia foram relatados em outros tipos de cirurgias da região genital, como foi visto em um estudo transversal ${ }^{2}$ com 102 pacientes submetidas à correção de fístula obstétrica, em que 22,5\% relataram não conseguir manter relações sexuais vaginais com penetração, 58,8\% relataram realização reduzida do orgasmo e 11,8\% relataram anorgasmia após a realização da cirurgia. Outro estudo ${ }^{3}$, dessa vez uma coorte inicial, analisando os resultados da genitoplastia em 6 pacientes com hiperplasia adrenal congênita, revelou que todas as mulheres tiveram resultados altamente anormais para sensação no clitóris e indicaram dificuldades sexuais e anorgasmia.

Apesar de incomum, encontrou-se um relato de caso $^{4}$ indicando que a embolização da artéria uterina tem potencial de causar anorgasmia temporária ou permanente dependendo da extensão da embolização e do fluxo colateral.

Quanto aos efeitos colaterais de medicamentos e a anorgasmia, percebeu-se prevalência dos antidepressivos e anticonvulsivantes e presença menos expressiva dos antipsicóticos.

Em um ensaio clínico randomizado duplo-cego ${ }^{5}$ que comparou a paroxetina (Inibidor Seletivo da Recaptação de Serotonina - ISRS) e o escitalopram (ISRS) para o tratamento de ansiedade generalizada, obteve como resultado, após um seguimento de duas semanas, que a anorgasmia foi um efeito colateral mais expressivo da paroxetina em relação ao escitalopram (26.2\% vs. 5.9\%), assim como a diminuição da libido (22.6\% vs. 4.9\%). Ainda em relação ao escitalopram, este fármaco, em um estudo duplo cego randomizado ${ }^{6}$, foi comparado com a reboxetina (Inibidor Seletivo da Recaptação de Noradrenalina - ISRN), ambos possuem efeito antidepressivo satisfatório, no entanto, o escitalopram apresentou mais efeitos colaterais em relação à função sexual, sendo a anorgasmia relatada por 5,9\% das mulheres sexualmente ativas no grupo da reboxetina vs 39\% no grupo do escitalopram. 
Um outro antidepressivo, a fluoxetina (ISRS), quando utilizada para o tratamento da síndrome pré menstrual, foi analisada em um estudo transversal ${ }^{7}$ com 30 mulheres, das quais $80 \%$ experimentaram efeitos colaterais sexuais negativos ou adversos, 53,3\% tiveram um efeito adverso na intensidade do orgasmo, 43,3\% tiveram um efeito adverso na duração do orgasmo e 10\% relataram anorgasmia.

Outra classe de fármacos que apresentou efeitos expressivos em relação à anorgasmia e à disfunção sexual foi a dos anticonvulsivantes, incluindo seus principais representantes, a Gabapentina e o Topiramato. Todos os estudos em relação a essa classe farmacológica incluídos na análise foram relatos de $\operatorname{casos}^{8,9}$, sendo um deles com 7 pacientes entre 40 e 62 anos que desenvolveram anorgasmia após serem medicados para enxaqueca com topiramato em doses variando entre 45 e $200 \mathrm{mg}$ ao dia. A resolução da anorgasmia foi obtida com redução ou descontinuação do medicamento. Tais efeitos mencionados também foram descritos em outros dois relatos de caso, reforçando os resultados encontrados. Repercussões semelhantes foram encontradas em dois relatos de casos envolvendo o uso da gabapentina, com melhora da anorgasmia com redução ou retirada ${ }^{(10,11,12)}$.

Um único relato de caso $^{13}$ foi encontrado relacionando o uso de antipsicóticos (clozapina, risperidona, olanzapina) com a anorgasmia. Tal efeito se dá devido ao bloqueio do receptor de dopamina na via tuberoinfundibular, o que leva à hiperprolactinemia, e esta pode causar anorgasmia, assim como diversos outros efeitos. Houve melhora com a troca dos medicamentos pela quetiapina. Também em um único relato de caso $^{14}$, foi observada a associação entre a anorgasmia e o implante hormonal de etonogestrel em uma paciente na ausência de outros comemorativos. Dois tratamentos foram implementados na tentativa de reverter o caso, a bupropiona $150 \mathrm{mg}$ por 4 semanas e, posteriormente, o sildenafil 50mg antes das relações sexuais por 2 semanas, no entanto, apenas a remoção do implante foi eficaz, demonstrada através do Female Sexual Function Index (FSFI). Tal associação, segundo o estudo, é um evento raro, visto que é o reflexo da falta de dados publicados, dificultando a estimativa da sua real prevalência.

Levando em consideração que a anorgasmia é um importante sintoma que interfere na saúde sexual das mulheres, o conhecimento do tratamento deve ser difundido na prática clínica, e este inclui tanto as abordagens medicamentosas quanto as não medicamentosas.

Em relação ao tratamento medicamentoso, duas classes farmacológicas foram descritas no manejo da anorgasmia, dentre elas, os agentes inibidores das fosfodiesterases e anti-histamínicos de primeira geração. Em dois relatos de caso ${ }^{15,16}$, o sildenafil e o vardenafil se mostraram eficazes para a reversão da anorgasmia nas doses de $100 \mathrm{mg}$ e $10 \mathrm{mg}$, respectivamente. E em uma série de quatro $\operatorname{casos}^{17}$, a ciproeptadina (anti-histamínico de $1^{\text {a }}$ geração) administrada em pacientes em uso de ISRS 
demonstrou-se capaz de reduzir ou reverter a anorgasmia causada pelos medicamentos, apresentando efeitos colaterais de sonolência e sedação em uma delas.

Ainda no âmbito do tratamento, um estudo randomizado $^{18}$ com 57 pacientes histerectomizadas comparou a terapia com estrogênio pelas vias oral e tópica. Observou-se melhora significativa da anorgasmia em ambos os grupos $(p<0,05)$, o que não pode ser visto em outros domínios como a taxa de baixa libido e frequência coital, que não foram estatisticamente significativos $(\mathrm{p}>0,05)$.

O sexo é um ato carregado de um teor emocional que ocorre em um contexto psicológico e cultural, o que justifica a adoção de abordagens não farmacológicas, como terapias sexuais e psicológicas, e outros métodos integrativos. Um estudo caso-controle ${ }^{19}$ com 47 casais heterossexuais demonstrou que problemas na comunicação do casal foram mais evidentes no grupo anorgásmico, visto que mulheres deste grupo apresentaram menos auto-aceitação sexual $(\mathrm{p}<0,001)$, além disso, relataram obter significativamente menos prazer em atividades sexuais quando comparadas às mulheres do grupo controle $(\mathrm{p}<0,001)$.

Além da abordagem tradicional, é importante ao médico saber identificar quando o encaminhamento interdisciplinar se faz necessário, como demonstrou um estudo ${ }^{20}$ que analisa dados referentes a 822 mulheres e 813 homens em que 44\% destes foram encaminhados por um médico, dentre as queixas apresentadas, a anorgasmia apareceu em 20,9\% dos casos. Ademais, a partir da análise dos dados obtidos, o estudo sugere que o problema sexual é uma manifestação física de problemas psicológicos ocultos ou de problemas de relacionamento, demonstrando a importância da contribuição psicossexual.

Reforçando a hipótese mencionada anteriormente, outro estudo ${ }^{21}$, que randomizou 65 mulheres com dificuldade de atingir o orgasmo em dois grupos de terapia cognitiva, uma delas baseada em mindfulness e, a outra, em terapia cognitiva comportamental, demonstrou que ambas as abordagens apresentaram aumento da função sexual, bem como aumento do desejo, do orgasmo e da satisfação sexual.

Um outro método de terapia, exclusivamente sexual, o método Betty Dodson, foi estudado em um ensaio clínico ${ }^{22}$ com 500 mulheres com anorgasmia, que comprovou a eficácia da abordagem, uma vez que $93 \%$ das pacientes tiveram um orgasmo durante o curso que foi testemunhado pelo terapêuta. Os resultados indicam que a etiologia da disfunção orgástica é um distúrbio do desenvolvimento psicossexual feminino, pois tanto mulheres na menopausa quanto mulheres em idade fértil com anorgasmia conseguiram atingir um orgasmo.

Como método alternativo, um ensaio clínico randomizado ${ }^{23}$ com 36 participantes estudou o uso de Tribulus terrestris (fitoterápico), como tratamento para hipoatividade sexual feminina, e os resultados mostraram melhora significativa do desejo $(p<0,01)$, excitação / lubrificação $(p=0,02)$, 
dor $(\mathrm{p}=0,02)$ e anorgasmia $(\mathrm{p}<0,01)$ em mulheres que usaram Tribulus terrestris, enquanto nenhuma melhora foi observada no grupo placebo $(\mathrm{p}>0,05)$. 


\section{DISCUSSÃO}

A anorgasmia é uma queixa que vai além da parte fisiológica do indivíduo, tendo, assim, diversas causas e tratamentos. Desta forma, é importante para o profissional que se depare com paciente acometido por este sintoma compreender a origem do problema para uma maior eficácia do tratamento. Logo, uma triagem minuciosa e um exame físico detalhado são os pilares para compreensão e abordagem da queixa. Esta última constatação pode ser enfatizada por um estudo ${ }^{24}$ realizado através de um questionário aplicado em residentes de ginecologia e obstetrícia, em que apenas 19,6\% relataram fazer o rastreamento das mulheres para problemas de função sexual, demonstrando uma necessidade de atividades didáticas baseadas em evidências para aumentar o conhecimento e confiança destes profissionais em relação a problemas de função sexual.

Como mencionado nos resultados, diversos são os fatores que influenciam diretamente a anorgasmia, sendo eles: cirurgias, medicamentos e fatores psicossociais. Em relação às cirurgias, a correção de prolapso de órgãos pélvicos demonstrou melhora da função sexual, enquanto a piora ocorreu após a correção de fístula obstétrica e genitoplastia. Diante do exposto, vê-se que a manipulação cirúrgica da área pélvica-abdominal pode gerar resultados diferentes em relação à função sexual, o que exige uma abordagem individualizada de cada caso.

O domínio do conhecimento de efeitos colaterais de medicamentos é de grande importância na prática clínica, já que estes podem causar sintomas que afetam a qualidade de vida, como é o caso da anorgasmia, em que foi visto que as principais classes farmacológicas relacionadas à essa queixa foram os antidepressivos, anticonvulsivantes, antipsicóticos e, raramente, o implante hormonal. Dessa forma, uma anamnese detalhada é essencial para identificar a origem da queixa, questionando acerca de medicamentos tomados e início dos sintomas, a fim de encontrar uma possível associação. 


\section{CONCLUSÃO}

Em suma, percebemos que a anorgasmia é uma afecção de múltiplos fatores que envolvem tanto o aspecto da relação da paciente consigo mesma e da relação com o seu parceiro, como efeitos colaterais de medicamentos e intervenções cirúrgicas. Observamos que esse conhecimento na prática clínica orienta abordagens precoces e eficazes para tratar a anorgasmia e assim promover qualidade de vida. Portanto, baseado em evidências, os possíveis tratamentos que se demonstraram satisfatórios foram a implementação dos agentes inibidores da fosfodiesterase, anti-histamínicos de primeira geração, uso de terapia estrogênica e fitoterápicos associados ao encaminhamento interdisciplinar.

A intenção do presente estudo não foi de esgotar todos os tratamentos possíveis para a anorgasmia, mas sim de compreender de maneira generalizada como o médico pode atuar em relação à queixa da paciente e quando encaminhar para uma abordagem psicossocial. A anorgasmia não é um sintoma que põe em risco a vida das mulheres, mas certamente é um fator que interfere na qualidade de vida e na relação conjugal com seu parceiro (a). 


\section{REFERÊNCIAS BIBLIOGRÁFICAS}

1. GEYNISMAN-TAN, Julia; KENTON, Kimberly; KOMAR, Alix; COLLINS, Sarah; LEWICKY-GAUPP, Christina; MUELLER, Margaret G.. Recovering sexual satisfaction after prolapse surgery: a secondary analysis of surgical recovery. International Urogynecology Journal, [S.L.], v. 29, n. 11, p. 1675-1680, 30 jun. 2018. Springer Science and Business Media LLC. http://dx.doi.org/10.1007/s00192-018-3690-7.

2. ANZAKU, Stephen A.; LENGMANG, Sunday J.; MIKAH, Samaila; SHEPHARD, Steven N.; EDEM, Bassey E.. Sexual activity among Nigerian women following successful obstetric fistula repair. International Journal Of Gynecology \& Obstetrics, [S.L.], v. 137, n. 1, p. 67-71, 17 jan. 2017. Wiley. http://dx.doi.org/10.1002/ijgo.12083.

3. Crouch NS, Minto CL, Laio LM, Woodhouse CR, Creighton SM. Genital sensation after feminizing genitoplasty for congenital adrenal hyperplasia: a pilot study. BJU Int. 2004 Jan;93(1):135-8. doi: 10.1111/j.1464-410x.2004.04572.x. PMID: 14678385.

4. Speir E, Shekhani H, Peters G. Temporary Anorgasmia Following Uterine Artery Embolization for Symptomatic Uterine Fibroids. Cardiovasc Intervent Radiol. 2017;40(11):1792-5.

5. Bielski RJ, Bose A, Chang CC. A double-blind comparison of escitalopram and paroxetine in the long-term treatment of generalized anxiety disorder. Ann Clin Psychiatry. 2005 Apr-Jun;17(2):65-9. doi: 10.1080/10401230590932326. PMID: 16075658.

6. Langworth S, Bodlund O, Ågren H. Efficacy and tolerability of reboxetine compared with citalopram: A double-blind study in patients with major depressive disorder. J Clin Psychopharmacol. 2006;26(2):121-7.

7. Oláh KS. The use of fluoxetine (Prozac) in premenstrual syndrome: is the incidence of sexual dysfunction and anorgasmia acceptable? J Obstet Gynaecol. 2002 Jan;22(1):81-3. doi: 10.1080/01443610120101808. PMID: 12521737.

8. Coebergh JA, Waldinger MD. Reversible anorgasmia with topiramate for migraine prophylaxis. J Neuropsychiatry Clin Neurosci. 2012;24(2):30-1. 
9. Sun C, Lay C, Broner S, Silberstein S, Tepper S, Newman L. Reversible anorgasmia with topiramate therapy for headache: a report of 7 patients. Headache. 2006 Oct;46(9):1450-3. doi: 10.1111/j.1526-4610.2006.00587.x. PMID: 17040342.

10. Mu M. Clinical / Scientific Notes Gabapentin-induced anorgasmia in women. Neurology. $2005 ; 115-20$.

11. Report C. Letters to the Editor Anorgasmia and Withdrawal Syndrome in a Woman Taking Gabapentin. :125-32.

12. Report C. Letters to the Editor Anorgasmia and Withdrawal

13. Keler R, Mongini F. Switch to quetiapine in antipsychotic agent-related hyperprolactinemia. Neurol Sci. 2002;23(5):233-5.

14. CHAPA, Hector O.; RAMIREZ, Alexiya; DAWSON, Daniel. Etonogestrel contraceptive implant-associated secondary anorgasmia. Contraception, [S.L.], v. 96, n. 4, p. 254-256, out. 2017. Elsevier BV. http://dx.doi.org/10.1016/j.contraception.2017.06.008.

15. Perelman MA. FSD partner issues: Expanding sex therapy with sildenafil. J Sex Marital Ther. 2002;28(SUPPL. 1):195-204.

16. ASHTON, Adam Keller. Vardenafil Reversal of Female Anorgasmia. American Journal Of Psychiatry, [S.L.], v. 161, n. 11, p. 2133-2133, nov. 2004. American Psychiatric Association Publishing. http://dx.doi.org/10.1176/appi.ajp.161.11.2133.

17. JAVANBAKHT, Arash. As-Needed Use of Cyproheptadine for Treatment of Selective Serotonin Reuptake Inhibitor-Related Female Anorgasmia. Journal Of Clinical Psychopharmacology, [S.L.], v. 35, n. 1, p. 91-93, fev. 2015. Ovid Technologies (Wolters Kluwer Health). http://dx.doi.org/10.1097/jep.0000000000000260. 
18. Long CY, Liu CM, Hsu SC, Wu CH, Wang CL, Tsai EM. A randomized comparative study of the effects of oral and topical estrogen therapy on the vaginal vascularization and sexual function in hysterectomized postmenopausal women. Menopause. 2006;13(5):737-43.

19. P. Kelly Mary, et al. Communication and Associated Relationship Issues in Female Anorgasmia. Journal of Sex \& Marital Therapy [Internet]. 2010 Aug 12 [cited 2021 Jun 24];30(4):263-276. DOI 10.1080/00926230490422403. Available from: https://www.tandfonline.com/doi/abs/10.1080/00926230490422403

20. Bronner G, Etran E, Schachter B, Lavee S, Galin A, Raviv G. [Contribution of psychosexual approach to assessment and treatment of sexual dysfunction: a working model]. Harefuah. 2009 Sep;148(9):595-9, 658. Hebrew. PMID: 20070048.

21. Adam F, De Sutter P, Day J, Grimm E. A Randomized Study Comparing Video-Based Mindfulness-Based Cognitive Therapy With Video-Based Traditional Cognitive Behavioral Therapy in a Sample of Women Struggling to Achieve Orgasm. J Sex Med. 2020 Feb;17(2):312-324. doi: 10.1016/j.jsxm.2019.10.022. Epub 2019 Dec 7. PMID: 31818723.

22. Struck P, Ventegodt S. Clinical holistic medicine: Teaching orgasm for females with chronic anorgasmia using the Betty Dodson method. ScientificWorldJournal. 2008;8:883-95.

23. Zanolla Dias de Souza Karla, et al. Efficacy of Tribulus terrestris for the treatment of hypoactive sexual desire disorder in postmenopausal women: a randomized, double-blinded, placebo-controlled trial. The Journal of the North American Menopause Society [Internet]. 2016 Nov 23 [cited 2021 Jun 24];23(11):1252-1256. DOI 10.1097/GME.0000000000000766. Available from: https://journals.lww.com/menopausejournal/Abstract/2016/11000/Efficacy of Tribulus terr estris for the treatment.16.aspx

24. Pancholy Apurva B, et al. Resident education and training in female sexuality: results of a national survey. The Journal of sex medicine [Internet]. 2010 Dec 08 [cited 2021 Jun 30]:361-6. DOI 10.1111/j.1743-6109.2010.02117.x. Available from: https://www.jsm.jsexmed.org/article/S1743-6095(15)33411-1/fulltext 